\title{
qsort R package: A New Tool for Scoring Q-sort Data
}

\section{DOI: $10.21105 /$ joss.00911}

\section{Software}

- Review ¿

- Repository c

- Archive ca

Submitted: 19 August 2018

Published: 02 December 2018

\section{License}

Authors of papers retain copyright and release the work under a Creative Commons Attribution $4.0 \mathrm{ln}$ ternational License (CC-BY).

\section{David N. Sousa ${ }^{1}$ and João R. Daniel ${ }^{1}$}

1 William James Center for Research, ISPA - Instituto Universitário

\section{Summary}

The use of Q-sets to describe subjective views on a specific research domain, in a way suitable for quantitative data analysis, has a long tradition in developmental psychology research. In this field, Q-sets generally consist of a large set of items, usually sentences printed on separate cards, that describe personality and behavioral characteristics. These items are sorted into a different number of numerical categories (e.g., 9) based on their salience as descriptors of an individual (e.g., 1- most uncharacteristic to 9- most characteristic). An individual Q-sort (i.e., the scores of all items descriptive of an individual) can then be compared (correlated) with criteria Q-sorts (description of a hypothetical person at the extreme high end of a domain) to index a score for a particular domain. Also, subsets of items can be averaged to derive a scale score (J. Block, 1961, J. H. Block \& Block (1969), J. H. Block \& Block (1980), John, Montgomery, \& Tate (2014), Vaughn, Santos, \& Coppola (2014)). In this paper we present an R package that computes scores from criteria Q-sorts and item scales as traditionally used in developmental psychology research.

The qsort package includes descriptions and scoring procedures for four commonly used Q-sets: Attachment Q-set (version 3.0) (Waters, 1995), California Child Q-set (J. H. Block \& Block, 1969), Maternal Behaviour Q-set (version 3.1) (Pederson, Moran, \& Bento, 1999), and Preschool Q-set (Baumrind, 1968) revised by Wanda Bronson).

The package contains seven objects:

- ex_qsort - example Q-sort datasets for the four different Q-sets: aqs, ccq, mbqs, pq.

- qsets_aqs, qsets_ccq, qsets_mbqs, and qsets_pq - four different datasets containing items descriptions, criteria socres and/or scales for the corresponding Q-set.

- qsort_score - a function to compute scores for Q-sort data using the criteria scores and/or scales from the qsets datasets.

- print_cards - a function to create a .pdf file with items' descriptions printed in separate cards.

Each Q-set dataset includes: (1) a column with item numbers, (2) a column with Qset initials, (3) a column with item description, (4) plus a variable number of columns according to available criteria sorts and scales. The aqs dataset includes: (1) security criterion scores, (2) dependency criterion scores, (3) (Pederson \& Moran, 1995) scales; compliance, secure base, fussy/difficult, enjoys physical contact, affective sharing, (4) and (Posada, Waters, Crowell, \& Lay, 1995) scales; smooth interactions with mother, physical contact with mother, interactions with other adults, proximity to mother. The ccq dataset includes scores of five criteria Q-sorts: (1) social competence criterion scores (2) self-esteem criterion scores (3) ego-resiliency criterion scores, (4) ego-control criterion scores, (5) social desirability criterion scores, and (6) emotion regulation scale scores retrieved from [shields1997emotion]. Ego-resiliency and ego-control criteria scores data for this dataset was retrieved from (Waters, Noyes, Vaughn, \& Ricks, 1985) and from Brian Vaughn (personal communication). The mbqs dataset includes scores of one criterion 
Q-sort: (1) sensibility criterion scores. The data for this dataset was retrieved from (Pederson et al., 1999). Finally the pq dataset includes scores of three criteria Q-sorts: (1) social competence, (2) self-esteem, and (3) social desirability. The data for this dataset was retrieved from (Waters et al., 1985).

(Waters et al., 1985) analysis of the discriminant validity of related constructs in the California Child Q-set (J. H. Block \& Block, 1969) and the Preschool Q-set (Baumrind, 1968) revised by Wanda Bronson) indicate that social desirability scores should be used to control for response bias when computing participants' scores (correlations) from criterion scores. Response bias in Q-sorts can occur when observers, confronted with two equally descriptive items of a participant, place one of the items higher (or lower) in the sort because it is more (or less) socially acceptable. Thus, the authors state that participant's scores are best indexed by a partial correlation between her/his Q-sort description and the criterion Q-sort, controlling for social desirability. The qsets_ccq and qsets_pq datasets include social desirability criterion scores. For these two Q-sets (ccq and pq) the qsort_score function will compute partial correlations between individual Q-sorts and criteria Q-sorts' scores, controlling for social desirability.

The following installs and loads qsort package:

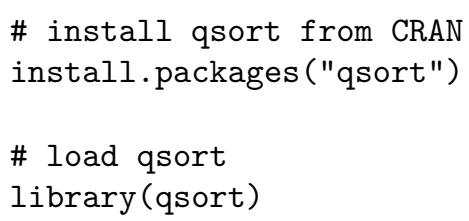

\section{Examples}

The qsort_score function uses the criteria scores and/or scales from the qsets datasets to compute scores for Q-sort data. The following applies the qsort_score function to ex_qsort\$ccq and stores the output data frame in data_ccq.

$R>$ data_ccq <- qsort_score(ex_qsort $\$ c c q$, qset_ccq, qsort_length $=100$, item1 = "ccq1", subj_id = "participant", group_id = "classroom")

$R>$ data_ccq

\begin{tabular}{|c|c|c|c|c|c|c|}
\hline 1 & 1 & -0.074 & -0.093 & -0.145 & $-0.0 \overline{12}$ & -0.137 \\
\hline 2 & 1 & -0.023 & 0.008 & 0.090 & 0.157 & 0.053 \\
\hline 3 & 1 & 0.092 & 0.086 & 0.112 & -0.021 & 0.132 \\
\hline 4 & 1 & -0.105 & -0.113 & -0.182 & -0.128 & -0.160 \\
\hline 5 & 1 & -0.010 & -0.039 & -0.092 & -0.092 & -0.053 \\
\hline 6 & 2 & -0.104 & -0.079 & -0.042 & 0.156 & -0.089 \\
\hline 7 & 2 & 0.051 & 0.079 & 0.168 & 0.169 & 0.124 \\
\hline 8 & 2 & 0.049 & 0.066 & 0.153 & 0.185 & 0.118 \\
\hline 9 & 2 & -0.024 & -0.007 & 0.009 & -0.026 & 0.007 \\
\hline 10 & 2 & -0.039 & -0.046 & -0.033 & 0.098 & -0.042 \\
\hline scomp_c & $v_{10}$ & _sest_c & c part & egores_c & partia & egocont_c \\
\hline 0.113 & & 0.047 & & -0.054 & & -0.007 \\
\hline-0.160 & & -0.073 & & 0.086 & & 0.155 \\
\hline-0.061 & & -0.053 & & -0.002 & & -0.026 \\
\hline 0.088 & & 0.047 & & -0.089 & & -0.123 \\
\hline 0.085 & & 0.012 & & -0.090 & & -0.090 \\
\hline-0.054 & & -0.004 & & 0.068 & & 0.160 \\
\hline
\end{tabular}




$\begin{array}{lrrrr}7 & -0.139 & -0.053 & 0.121 & 0.166 \\ 8 & -0.130 & -0.068 & 0.101 & 0.182 \\ 9 & -0.069 & -0.026 & 0.004 & -0.026 \\ 10 & -0.003 & -0.019 & 0.005 & 0.100 \\ & \text { shields_s_emreg } & & & \\ 1 & 4.3 & & & \\ 2 & 5.9 & & \\ 3 & 5.1 & & \\ 4 & 3.7 & & \\ 5 & 4.4 & & \\ 6 & 5.7 & & \\ 7 & 5.9 & & \\ 8 & 5.9 & & & \\ 9 & 5.0 & & & \\ 10 & 5.2 & & & \end{array}$

For details about the qsort_score function usage type ?qsort_score. The first two columns of data_ccq correspond to qset, participant and classroom identification variables; the remaining columns refer to correlation values computed from correlating individual Q-sorts with the criteria Q-sorts' scores (present in qsets_ccq). The names of these columns follow the column names in qsets_ccq datasets (e.g., social competencescomp_c, self-esteem- sest_c, etc.). Variable names are detailed in the help files of the different qsets. Scores obtained from partial correlations controlled by social desirability correspond to columns in the output data frame with names starting with partial

To create a .pdf file where items' descriptions are printed in separate cards, the print_cards function can be used as in the following example:

R> print_cards(qset $=$ "aqs", desc_col = "description", dir.print = getwd () )

This creates a .pdf file, in in the working directory, with Attachment Q-set item cards.

For further details on how to use the functions provided and the Q-set datasets contained in the qsort package type help(qsort). At the moment, qsort package only analyses data for four different Q-sets, but it can be easily updated to extend its capabilities to other Q-sets scored from scales or criteria scores. We believe this tool to be of great interest and utility for developmental psychology researchers and we invite them to contribute with new or updated versions of existing Q-sets, criterion scores and derived scales.

\section{References}

Baumrind, D. (1968). Manual for the preschool behaviour q-set. Parental research project. Berkeley, CA: Institute of Human Development, University of California.

Block, J. (1961). The q-sort method in personality assessment and psychiatric research. Springfield, IL: Charles C. Thomas.

Block, J. H., \& Block, J. (1969). The california child q-set. Berkeley, CA: Institute of Human Development.

Block, J. H., \& Block, J. (1980). The role of ego-control and ego-resiliency in the organization of behaviour. Hillsdale, NJ: Erlbaum.

John, A., Montgomery, D., \& Tate, A. L. H. (2014). Using q methodology in conducting research with young children. In O. N. Saracho (ed.), handbook of research methods in early childhood education: Review of research methodologies (pp. 147-173). Charlotte, NC: Information Age Publishing. 
Pederson, D. R., \& Moran, G. (1995). A categorical description of infant-mother relationships in the home and its relation to q-sort measures of infant-mother interaction. Monographs of the Society for Research in Child Development, 60 (2/3), 111-132. doi:doi:10.1111/j.1540-5834.1995.tb00207.x

Pederson, D. R., Moran, G., \& Bento, S. (1999). Maternal behaviour q-sort (version 3.1). London, ON: Psychology Department, Western University.

Posada, G., Waters, E., Crowell, J. A., \& Lay, K. (1995). Is it easier to use a secure mother as a secure base? Attachment q-sort correlates of the adult attachment interview. Monographs of the Society for Research in Child Development, 60 (2/3), 133-145. doi:doi:10.1111/j.1540-5834.1995.tb00208.x

Vaughn, B. E., Santos, A. J., \& Coppola, G. (2014). Q-methodology and q-sorting as tools for addressing research questions in educational settings: Historical overview and illustrations using three standardized q-sets. In O. N. Saracho (ed.), handbook of research methods in early childhood education: Review of research methodologies (pp. 175-202). Charlotte, NC: Information Age Publishing.

Waters, E. (1995). Appendix a: The attachment q-set (version 3. 0). Monographs of the Society for Research in Child Development, 60 (2/3), 234-246. doi:doi:10.1111/j.15405834.1995.tb00214.x

Waters, E., Noyes, D. M., Vaughn, B. E., \& Ricks, M. (1985). Q-sort definitions of social competence and self-esteem: Discriminant validity of related constructs in theory and data. Developmental Psychology, 60 (2/3), 508-522. doi:doi:10.1037/0012-1649.21.3.508 